\title{
Fatal perforation with subcutaneous emphysema complicating ERCP
}

An 80-year-old man was admitted for cholestatic jaundice. He had undergone Billroth II gastrectomy 32 years earlier due to an ulcer. Magnetic resonance cholangiopancreatography revealed choledocholithiasis. At endoscopic retrograde cholangiopancreatography (ERCP), reverse sphincterotomy, balloon dilation of the sphincterotomy, and bile duct stone removal were performed ( Fig. 1 and - Fig.2). Shortly afterwards the patient complained of epigastric pain accompanied by abdominal, thoracic, and supraclavicular crepitus. Computed tomography (CT) revealed significant subcutaneous emphysema, pneumomediastinum, left pneumothorax, pneumoperitoneum, retropneumoperitoneum, fluid collection in the abdomen and pelvis, and pleural effusion with atelectasis ( Fig. 3 and - Fig.4). Surgical intervention was not undertaken, due to the patient's comorbidities and poor performance status; he was treated with nasogastric suction, hydration, and antibiotics, but 1 day later became febrile. Emergency CT ( $\bullet$ Fig.5) showed reduction of the pneumoperitoneum, retropneumoperitoneum, pneumothorax, and subcutaneous emphysema, but an increase in the right pleural effusion and atelectasis. He gradually deteriorated and finally succumbed to his illness amidst respiratory distress and sepsis.

ERCP is widely used for the management of pancreatobiliary pathologies [1]. The most common cause of luminal air leakage at ERCP is duodenal perforation [2]. Less often, any site of reduced resistance (a diverticulum, ulcer, or tumor) may serve as a "release valve" during insufflation, even without obvious perforation $[3,4]$. In our case, air leakage became clinically apparent due to massive subcutaneous emphysema, after which CT established the diagnosis. However, in smaller leakages, clinical manifestations may be subtle; therefore the clinician may overlook this rare complication of ERCP. If perforation is suspected, conservative treatment may be discussed, but surgical intervention should be prompt once a perforation is diagnosed [5]. In our case, air leakage was most probably a result of microperforation and valve formation due to balloon dilation post sphincterot-
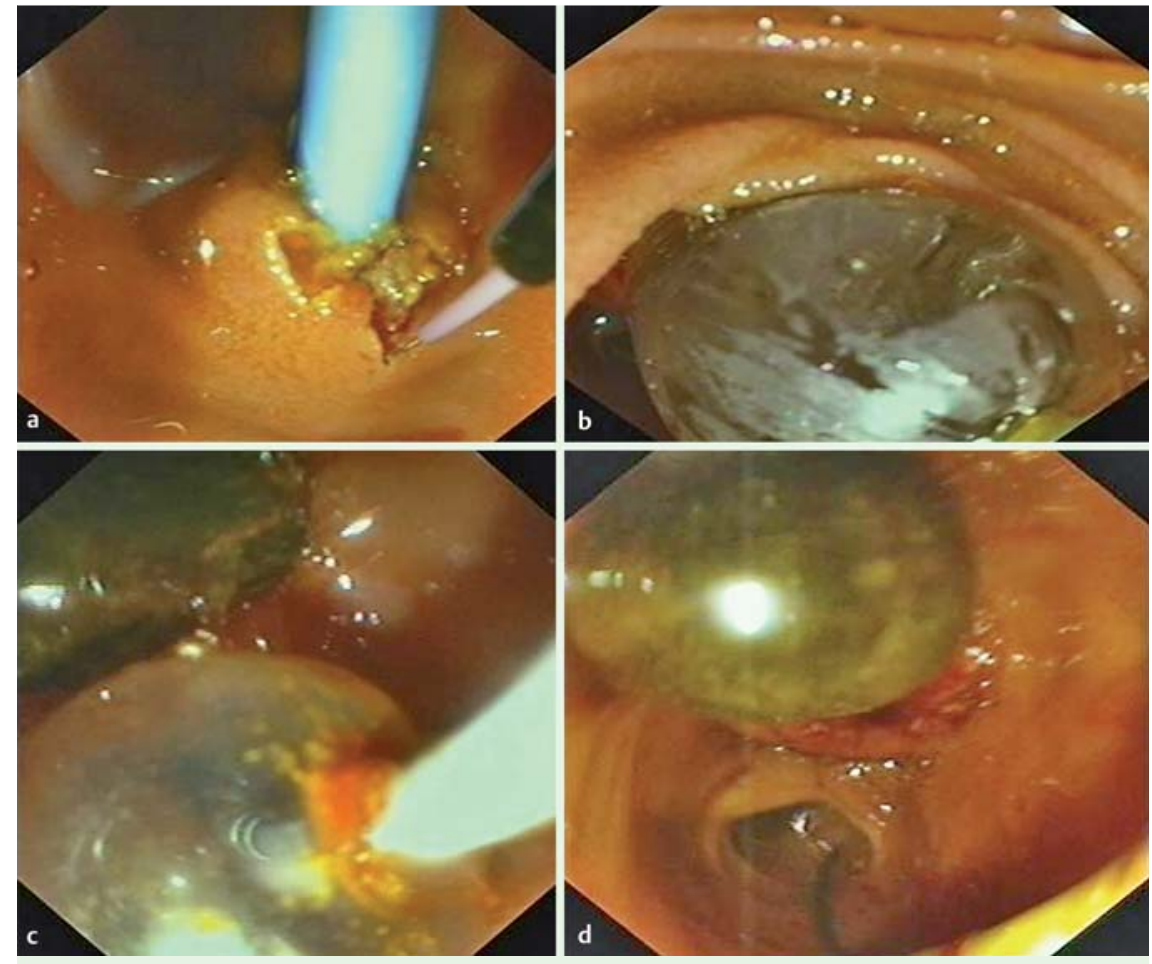

Fig. 1 Endoscopic retrograde cholangiopancreatography (ERCP): a reverse sphincterotomy, $\mathbf{b}$ balloon dilation, and $\mathbf{c}, \mathbf{d}$ extraction of a gallstone.

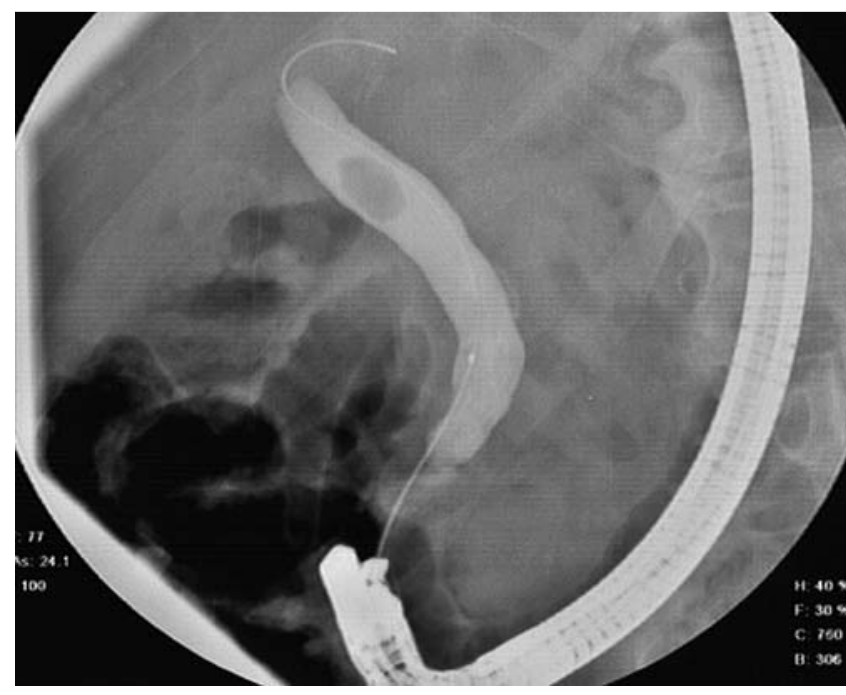

Fig. 2 Fluoroscopy during endoscopic retrograde cholangiopancreatography (ERCP), demonstrating an impacted bile duct stone.

omy, from the retroperitoneal space to the thorax via the diaphragmatic hiatuses, into the mediastinum, pleural space, and subcutaneous tissue.

\section{Endoscopy_UCTN_Code_CPL_1AK_2AC}

Competing interests: None 

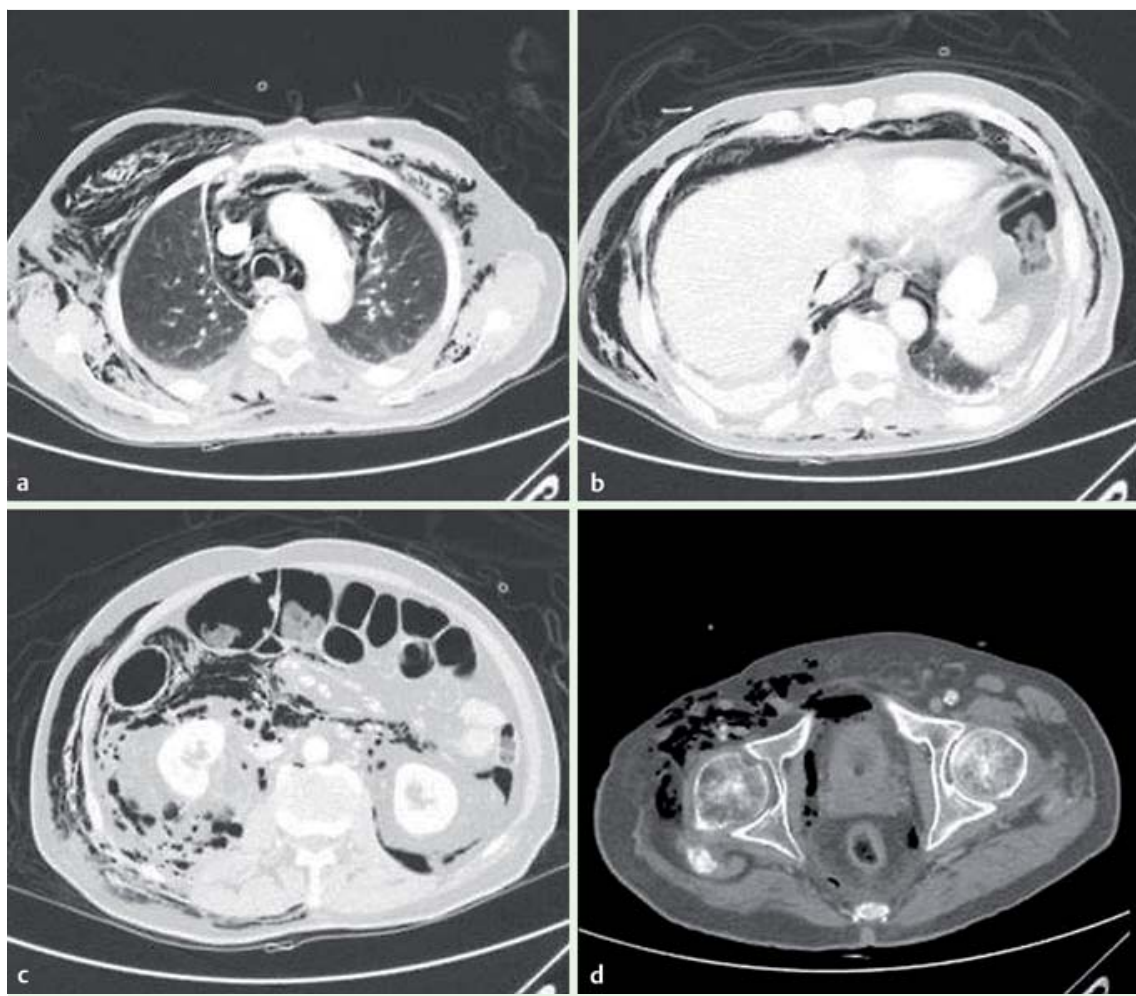

Fig. 3 Contrast-enhanced abdominal computed tomography (CT) obtained with a soft-tissue window shows free air in: a the mediastinum; $\mathbf{b}$ the subcutaneous tissues; $\mathbf{c}$ the peritoneal cavity, retroperitoneum, and subcutaneous tissues, $\mathbf{d}$ the peritoneal cavity and subcutaneous tissues.

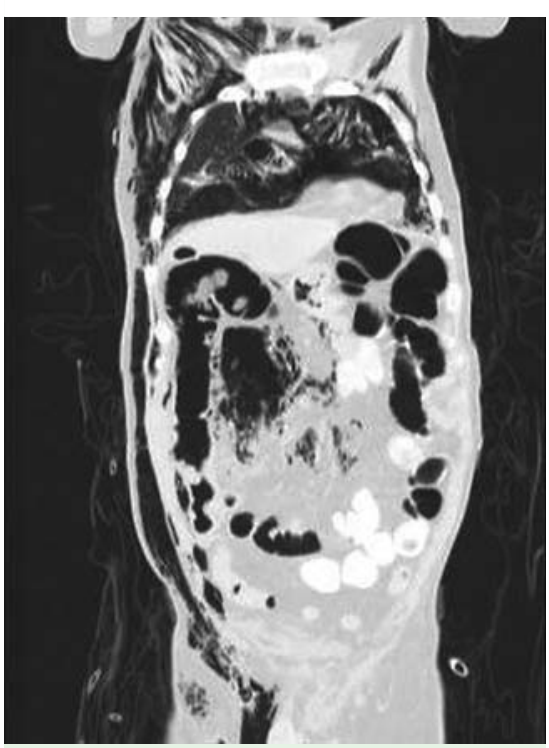

Fig. 4 A coronal multiplanar reconstruction shows a large amount of free air.

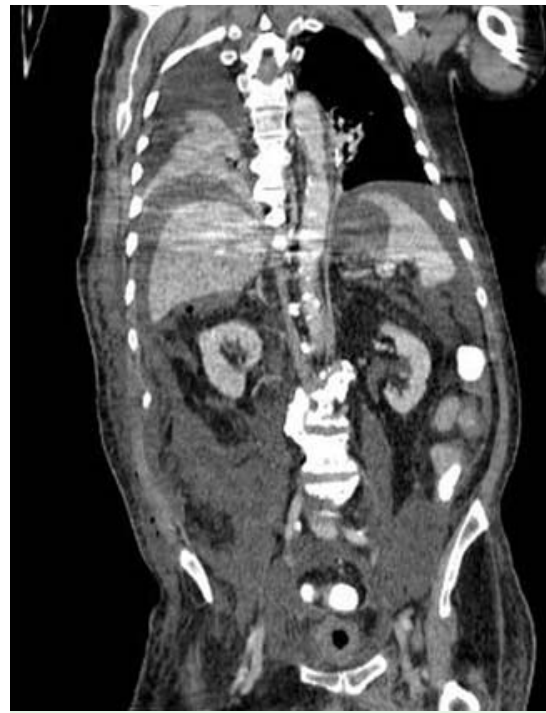

Fig. 5 Repeat computed tomography (CT) (coronal reconstruction) obtained 6 days after the earlier CT scan. There was a significant decrease in the amount of free air, but increased fluid diffusely in the peritoneal cavity and right pleural effusion.

\section{Beintaris ${ }^{1}$, D. Polymeros ${ }^{1}$,}

I. S. Papanikolaou' ${ }^{1}$ C. Kontopoulou ${ }^{2}$,

A. Kourikou ${ }^{1}$, A. D. Sioulas ${ }^{1}$, N. Danias ${ }^{3}$, G. Dimitriadis ${ }^{1}$, K. Triantafyllou ${ }^{1}$

${ }^{1}$ Hepatogastroenterology Unit, 2nd Department of Internal Medicine and Research Institute, Attikon University General Hospital, Medical School, University of Athens, Greece

2 2nd Department of Radiology, Attikon University General Hospital, Medical School, University of Athens, Greece 3 4th Department of Surgery, Attikon University General Hospital, Medical School, University of Athens, Greece

\section{References}

1 Loperfido S, Angelini G, Benedetti G et al. Major early complications from diagnostic and therapeutic ERCP: a prospective multicenter study. Gastrointest Endosc 1998; 48: 1-10

2 Fujii L, Lau A, Fleischer DE et al. Successful nonsurgical treatment of pneumomediastinum, pneumothorax, pneumoperitoneum, pneumoretroperitoneum, and subcutaneous emphysema following ERCP. Gastroenterol Res Pract 2010; 2010: 289135

3 Kocaman O, Sipahi M, Ubukc AC et al. Porous diaphragm syndrome after ERCP in a patient with bile duct stricture. Turk J Gastroenterol 2009; 20: 157-158

4 Mosler P, Fogel EL. Massive subcutaneous emphysema after attempted endoscopic retrograde cholangiopancreatography in a patient with a history of bariatric gastric bypass surgery. Endoscopy 2007; 39: 155

5 Stapfer M, Selby RR, Stain SC et al. Management of duodenal perforation after endoscopic retrograde cholangiopancreatography and sphincterotomy. Ann Surg 2000; 232: $191-198$

\section{Bibliography}

DOI http://dx.doi.org/

10.1055/s-0032-1309758

Endoscopy 2012; 44: E313-E314

(c) Georg Thieme Verlag KG

Stuttgart · New York

ISSN 0013-726X

\section{Corresponding author}

\section{S. Papanikolaou, MD}

\section{Hepatogastroenterology Unit}

2nd Department of Internal Medicine and

Research Institute

Attikon University General Hospital

Medical School, University of Athens

31 Vournazou Street

11521 Athens

Greece

Fax: +30-210-5326422

ispapn@hotmail.com 